Documentation et bibliothèques

\title{
La nouvelle carte du monde : bienvenue au $\mathrm{XXI}^{\mathrm{e}}$ siècle The New Map of the World: Welcome to the $21^{\text {st }}$ Century
}

\section{Jean-Louis Roy}

Volume 65, numéro 1, janvier-mars 2019

Actrices et acteurs de changement

URI : https://id.erudit.org/iderudit/1061814ar

DOI : https://doi.org/10.7202/1061814ar

Aller au sommaire du numéro

\section{Éditeur(s)}

Association pour l'avancement des sciences et des techniques de la documentation (ASTED)

\section{ISSN}

0315-2340 (imprimé)

2291-8949 (numérique)

\section{Découvrir la revue}

\section{Citer cet article}

Roy, J.-L. (2019). La nouvelle carte du monde : bienvenue au XXI ${ }^{\mathrm{e}}$ siècle. Documentation et bibliothèques, 65(1), 5-11. https://doi.org/10.7202/1061814ar

\section{Résumé de l'article}

" Notre monde est en plein travail d'enfantement », disait Virginia Woolf. Dans les institutions culturelles, les bibliothèques, les musées, et plus généralement dans tout temple de la connaissance, le changement opère, force multiforme qui transforme et modèle nos habitudes de travail, nos pratiques et les rapports que nous entretenons avec nos usagers. Comment évaluer et valoriser la place que nous occupons dans la société dite du savoir ? Comment concilier le changement avec des notions aussi capitales que la vérité, la solidarité culturelle et sociale, ainsi que l'échange, ce puissant catalyseur de la dignité humaine ?

Apprivoiser les flux de données pour rendre ces dernières fiables et intègres, s'approprier les nouvelles technologies afin de bâtir une offre de qualité libre et ouverte, autant de chantiers à relever pour celles et ceux dont la mission première est de constituer un thésaurus du savoir, véritable agora à la disposition de tous, accessible, sincère et altruiste.

Le changement que nous observons est issu de quatre mutations majeures dans l'histoire humaine : la transformation de la carte financière, économique et commerciale du monde; le déploiement de l'ère numérique qui change à tout jamais les rapports de l'être humain à la connaissance ; l'expansion démographique sur les continents asiatique et africain, qui bouleversera bientôt la répartition actuelle de la population mondiale ; enfin, les immenses défis environnementaux dont nos sociétés doivent se saisir sans plus tarder.

Des premières avancées de la société québécoise jusqu’à la création d'un laboratoire de renommée internationale en intelligence artificielle, l'histoire se tisse grâce à l'information que l'esprit humain expérimente comme matière première, et à laquelle il insuffle une énergie vitale. Le changement doit se poursuivre avec le partage de l'information sous toutes ses formes et auprès de tous, ce qui nécessite notamment de mettre fin à ce très vieux scandale qu'est l'illettrisme dans nos sociétés savantes et connectées. Cela demande aussi d'accélérer le passage à la civilisation numérique, par la mise en place du dépôt légal numérique, la numérisation des collections patrimoniales, la création de bibliothèques d'avant-garde qui font la part belle aux laboratoires d'innovation et de création, telle la future bibliothèque Saint-Sulpice.

Il nous faudra enfin porter notre réflexion sur l'avenir de la création : à l'âge de la robotique, des algorithmes, de l'intelligence artificielle et des données de masse, quel est donc l'avenir de la mémoire, de la production et du partage de la connaissance?
Tous droits réservés $@$ Association pour l'avancement des sciences et des techniques de la documentation (ASTED), 2019
Ce document est protégé par la loi sur le droit d'auteur. L’utilisation des services d'Érudit (y compris la reproduction) est assujettie à sa politique d'utilisation que vous pouvez consulter en ligne.

https://apropos.erudit.org/fr/usagers/politique-dutilisation/ 


\section{LA NOUVELLE CARTE DU MONDE: BIENVENUE AU XXI' SIÈCLE ${ }^{1}$}

Jean-Louis ROY

Président-directeur général de Bibliothèque et Archives nationales du Québec (BAnQ)

JL.Roy@banq.qc.ca

"Notre monde est en plein travail d'enfantement", disait Virginia Woolf. Dans les institutions culturelles, les bibliothèques, les musées, et plus généralement dans tout temple de la connaissance, le changement opère, force multiforme qui transforme et modèle nos habitudes de travail, nos pratiques et les rapports que nous entretenons avec nos usagers. Comment évaluer et valoriser la place que nous occupons dans la société dite du savoir? Comment concilier le changement avec des notions aussi capitales que la vérité, la solidarité culturelle et sociale, ainsi que l'échange, ce puissant catalyseur de la dignité humaine?

Apprivoiser les flux de données pour rendre ces dernières fiables et intègres, s'approprier les nouvelles technologies afin de bâtir une offre de qualité libre et ouverte, autant de chantiers à relever pour celles et ceux dont la mission première est de constituer un thésaurus du savoir, véritable agora à la disposition de tous, accessible, sincère et altruiste.

Le changement que nous observons est issu de quatre mutations majeures dans l'histoire humaine: la transformation de la carte financière, économique et commerciale du monde; le déploiement de l'ère numérique qui change à tout jamais les rapports de l'être humain à la connaissance; l'expansion démographique sur les continents asiatique et africain, qui bouleversera bientôt la répartition actuelle de la population mondiale; enfin, les immenses défis environnementaux dont nos sociétés doivent se saisir sans plus tarder.

Des premières avancées de la société québécoise jusqu'à la création d'un laboratoire de renommée internationale en intelligence artificielle, l'histoire se tisse grâce à l'information que l'esprit humain expérimente comme matière première, et à laquelle il insuffle une énergie vitale. Le changement doit se poursuivre avec le partage de l'information sous toutes ses formes et auprès de tous, ce qui nécessite notamment de mettre fin à ce très vieux scandale qu'est l'illettrisme dans nos sociétés savantes et connectées. Cela demande aussi d'accélérer le passage à la civilisation numérique, par la mise en place du dépôt légal numérique, la numérisation des collections patrimoniales, la création de bibliothèques d'avant-garde qui font la part belle aux laboratoires d'innovation et de création, telle la future bibliothèque Saint-Sulpice.

II nous faudra enfin porter notre réflexion sur l'avenir de la création: à l'âge de la robotique, des algorithmes, de l'intelligence artificielle et des données de masse, quel est donc l'avenir de la mémoire, de la production et du partage de la connaissance?

\section{The New Map of the World: Welcome to the $21^{\text {st }}$ Century}

As Virginia Woolf said, our world is in the midst of childbirth. Multifaceted change is underway in cultural institutions, libraries, museums and, generally, in all places where knowledge is created. Change is transforming our work habits, practices and relationships with our users. How do we evaluate and highlight our place in the knowledge society? How do we reconcile change with the important notions of truth, cultural and social solidarity as well as sharing, that forceful catalyst of human dignity?

Harnessing the mass of data to make them reliable and truthful and taking ownership of new technologies in order to build a free and open tender are part of the challenge to be met by those whose primary mission is to build a knowledge base, that is accessible, sincere, and altruistic for all.

The change we are currently experiencing is the result of four major events in human history: the transformation of the financial, economic and commercial map of the world; the unfolding of a digital era that has forever changed the relationship of human beings to knowledge; the demographic expansions in Asia and Africa that will soon disrupt the distribution of the world's population; and, finally, the colossal environmental issues that our society must immediately face.

Beginning with the first developments in Québec society and followed by an internationally renowned artificial intelligence laboratory, history is created with the information used by the human mind to which it imparts a vital energy. Change must be undertaken by sharing information in all its forms and with all, which means putting an end to the scandal that is illiteracy in our learned and connected societies. This also requires accelerating the transition to a digital civilisation, by establishing a digital legal deposit, the scanning of heritage collections and the creation of avant-garde libraries that provide a space for the innovative and creative laboratories such as the future SaintSulpice library.

We also have to think about the future of creation. In an era of robotics, algorithms, artificial intelligence, and big data, what does the future hold for the safeguard, the production and the sharing of knowledge?

1. Cet article est une adaptation de l'allocution de Jean-Louis Roy, PDG de BAnQ, au Congrès des professionnels et professionnelles de l'information, Actrices et acteurs de changement, qui s'est tenu à Montréal le 14 novembre 2018. 


\section{Actrices et acteurs de changement}

Ces trois derniers jours, les intervenantes et intervenants à notre congrès ont identifié en situation réelle - comme on dit en temps réel - les nécessités sociales, les évolutions technologiques et les exigences économiques qui inscrivent le changement dans l'exercice quotidien de nos professions. Ils nous ont rappelé que l'information, la documentation et le partage de données ne se résument pas à d'innombrables échanges mécaniques et éphémères, mais qu'ils sont le terreau de l'enrichissement continu des savoirs humains partagés et créés, de la responsabilité citoyenne déployée, de la conscience des filiations et des héritages qui, dans la longue durée du temps, a produit la liberté humaine et son double, l'égalité de toutes et de tous. J'emprunte à Marguerite Yourcenar (1980) l'idée que les échanges qui sont notre quotidien «relie[nt] l'homme à tout ce qui a été, et sera, et non pas à une mode d'un jour».

Vous n'êtes pas seulement des courtiers en connaissance, mais des guides, des partenaires, des sherpas, à un moment qui peut être décisif pour vos vis-à-vis, celles et ceux qui, dans l'immensité de ce qui est disponible, trouvent avec vous, grâce à vous, ce qu'ils recherchaient, ce qu'ils attendaient. Il y a de la transcendance dans cette rencontre des esprits, entre celui qui vous communique son besoin et le fait devenir vôtre, et vous dont l'esprit retourne vers celui qui a initié cet échange. Universel parce que constitutif, l'échange est un des plus grands phénomènes dans la vie de la famille humaine, dans la vie de chaque être humain. Imaginer un monde sans échanges, c'est imaginer un monde sans vie. La dignité humaine, l'éthique des personnes et des groupes, et donc la vie, n'existent que par l'échange, tant le sentiment de la dignité d'autrui est réciproque ou n'est pas. À cette aune, les bibliothèques sont des maisons de droits humains.

Je vous ai entendu nous dire qu'il faut penser «usager» et, en conséquence, rénover les services de référence, renouveler les relations des couples enseignants-bibliothécaires, entrepreneurs-bibliothécaires, usagers-bibliothécaires, s'ouvrir à de nouvelles pratiques dont les toolkit qui ont bonne presse chez nos voisins. Vous nous avez conviés à considérer la dimension sociale de nos services professionnels, du lien entre bibliothèque et développement local, bibliothèque et justice sociale, bibliothèque et communauté. Vous avez aussi plaidé pour que nous soyons attentifs aux évolutions des sciences de l'information, aux leviers technologiques innovants telles les commandes tactiles et la reconnaissance automatique. Vous nous avez invités à réfléchir au profil des bibliothèques du futur qui foisonnent de données de nouvelle génération et des interférences à venir de l'intelligence artificielle.

Enfin, la question maîtresse, celle qui commande à toutes les autres, celle qui se pose depuis les origines et qui ne cesse de s'éloigner dès que l'on s'en rapproche, celle qui se pose aujourd'hui avec une forte exigence, la question de la vérité, a été explorée par certains d'entre vous. Ceux-là ont évoqué la lutte contre les fake news, cette très ancienne démangeaison dont l'existence est aujourd'hui amplifiée par les technologies que vous savez, et qui constitue l'antithèse absolue de l'ADN de nos professions et de notre service public, tout entier dévoués à la recherche et à la défense de la vérité.

Il y a dans ces thèmes choisis et développés par vous ces derniers jours, une odeur de changement, l'idée d'un authentique passage, celle d'une révision des repères et d'une transformation des pratiques: ces chantiers multiples s'adressent à tous, qu'ils soient bibliothécaires, professionnels, théoriciens ou techniciens. Finalement, vos observations et vos propositions visent toutes à parfaire et à affermir l'échange, cette absolue nécessité dans la vie de la famille humaine, ce levier de la dignité humaine, comme nous l'avons écrit précédemment. Ce travail sur nous-mêmes n'est pas seulement de l'ordre des moyens. Spinoza (1955) avait raison d'affirmer que «le but de l'organisation en société, c'est la liberté».

"Le monde tout entier est en plein travail d'enfantement», selon le mot de Virginia Woolf (1937). Cela doit se traduire dans nos planifications stratégiques, nos formations continues, nos investissements de recherche, nos activités parallèles; autrement dit, dans nos contributions diverses au développement social et au partage de la connaissance. Pour ne pas être submergée par les flux de données découlant de cette gestation et par les puissances qui les incarnent avec des visées hégémoniques, notre offre, la vôtre et la nôtre, doit être constamment enrichie, rester libre, ouverte, hissée à la hauteur des besoins sociaux et culturels les mieux documentés, élevée au niveau des impératifs de la concurrence économique devenue mondiale et portée par les technologies les plus avancées.

Notre valeur ajoutée est tout entière contenue dans notre capacité à lier connaissance et développement d'une société donnée, historique, irréductible. Le Québec a besoin de ces investissements pour rester lui-même, maintenir et enrichir ses investissements sociaux et culturels, garantir et enrichir un niveau de vie convenable pour tous, ce qui n'est pas le cas présentement. Telles doivent être nos exigences envers nous-mêmes, nos partenaires, nos bailleurs de fonds, en conséquence des services qui sont attendus de nous. De nous, dans les administrations publiques, locales et nationales, qui ont besoin de données fiables et intègres pour une gouvernance éclairée, juste et efficace. De nous, dans les entreprises de production de biens et de services des secteurs public et privé, qui ont tout autant besoin de données fiables et intègres pour construire une offre de qualité capable de tenir sa place dans la compétition mondiale. De nous, dans les institutions de savoir, de la 
prématernelle au postdoctorat, sans oublier nos centres de recherche privés et publics qui ont aujourd'hui la redoutable mission de montrer le monde tel qu'il advient et non dans ses représentations périmées. De nous enfin, dans les bibliothèques publiques, de la plus modeste à la mieux dotée, véritables biens nationaux et parmi les dernières agoras encore accessibles pour tous nos concitoyens, mettant à la disposition de chacun et de tous un thésaurus de savoir, sans intérêts dissimulés, sans appropriation douteuse ni manipulation véreuse.

Voilà bien des motifs de nous réjouir de l'occasion qui nous est donnée de nous retrouver, afin notamment d'évaluer et de valoriser la place que nous occupons dans la société dite du savoir, le rôle qui est le nôtre dans les délibérations conduisant aux mises à jour de la gouvernance, à la création continue de la solidarité culturelle et sociale et à l'appropriation responsable de la science et de la technologie. Actrices et acteurs de changement: ce sont celles et ceux qui agissent, qui prennent et revendiquent une part active dans le changement. Vos contributions, que je viens d'évoquer, témoignent de ce désir d'agir, d'être partie prenante $\mathrm{du}$ changement. Comment en serait-il autrement? Ce monde, notre monde, est en pleine gestation. Actrices et acteurs de changements, vous dites! L'aventure qui est et sera la nôtre dans les années à venir est issue de quatre mutations sans précédent dans l'histoire de la famille humaine, quatre mutations qui appellent le changement.

\section{La nouvelle carte du monde}

Le basculement de la richesse de l'Ouest vers l'Est et le Sud de la planète a transformé à jamais la carte financière, économique et commerciale du monde. Il a fait de l'Asie son épicentre et de la Chine, anciennement le plus grand pays pauvre du monde, la deuxième puissance économique mondiale, la première peut-être demain. Ce bouleversement a également permis à près d'un milliard de personnes de sortir de la pauvreté.

Au tournant des millénaires s'est produit, selon les termes de l'OCDE (2010), le plus grand transfert financier, commercial et technologique de l'histoire de l'humanité - transfert de l'investissement direct étranger, délocalisation des entreprises du Nord et déplacement de la puissance financière. Ce n'est pas seulement la richesse, la science et la technologie qui ont été transférées vers l'Est et le Sud, mais bien la capacité durable de les produire. En conséquence, il nous faudra prêter une attention particulière aux contributions intellectuelles, culturelles et scientifiques du monde entier, et leur faire une place dans nos investigations, nos listes privilégiées et nos médiations. Il nous faut prendre acte de cette mutation qui va de pair avec une mutation technologique sans précédent dans l'histoire de l'humanité.

\section{Le déploiement de l'ère numérique}

Le déploiement de l'ère numérique et son emprise sur un nombre sans cesse croissant de domaines ont provoqué l'émergence de l'humanité numérique, composée aujourd'hui de 4,2 milliards d'internautes ${ }^{2}$ qui seront 5,5 milliards en 2025. Une ascension fulgurante pour une espèce quasi inexistante il y a 30 ans.

Cette révolution technique est unique pour deux raisons. Elle s'est déployée en temps réel dans la presque totalité des sociétés humaines et, contrairement aux précédentes révolutions technologiques qui démultipliaient les énergies matérielles de l'humanité, la révolution numérique, elle, démultiplie ses énergies cognitives et change à tout jamais les rapports de l'homme à la connaissance, qui est notre matière première.

La croissance des plateformes du numérique et le transfert des activités humaines du monde historique vers le monde numérique sont continus. On a estimé en 2016 que seulement $8 \%$ des activités humaines susceptibles d'être transférées l'ont été effectivement et on anticipe une accélération exponentielle de ce transfert. Toutes les projections annoncent des croissances considérables en raison notamment du passage à la $5 \mathrm{G}$ et à l'Internet des objets. On évoque 80 milliards d'objets connectés en 2025, contre 15 milliards aujourd'hui. Alors, les trois grandes fonctionnalités de l'Internet des objets se feront sentir: le suivi des consommations diverses, la consultation des dossiers médicaux et l'évolution de l'interaction entre les personnes (en permettant aux appareils d'échanger des données sur les activités humaines).

\section{L'enrichissement démographique}

Enfin, on ne peut prétendre comprendre quoi que ce soit aux enjeux actuels et à venir au niveau mondial si on ne prend pas en compte l'expansion démographique en cours. D'ici 2040, plus de 2 milliards de personnes naîtront sur les continents asiatique et africain, ce qui bouleversera la répartition de la population mondiale telle qu'elle s'est déployée depuis des siècles et consacrera définitivement la prépondérance de ces continents dans les affaires du monde. Y vivra, au milieu de ce siècle, près de $80 \%$ de la population mondiale, tandis que les $20 \%$ restants vivront en Europe - incluant la Fédération de Russie - et sur le continent américain. Ces $20 \%$ représenteront une population vieillissante, alors que l'Afrique et l'Inde incarneront la jeunesse du monde. Autour de 2050, un homme sur quatre sera africain.

\footnotetext{
2. 5,1 milliards de personnes disposent d'un téléphone mobile, dont la majorité sont des téléphones intelligents. Plus de 3 milliards de personnes utilisent les réseaux sociaux.
} 


\section{Les défis environnementaux}

En ayant conscience de traiter trop rapidement la question de l'indispensable reconnexion de l'homme et de la nature, je rappellerai deux statistiques qui font consensus : $60 \%$ des espèces animales sauvages ont disparu depuis 40 ans, et si aucune solution n'est trouvée dans ce domaine, il y aura 200 millions de réfugiés climatiques en 2050.

Être actrice et acteur de changement aujourd'hui, c'est appréhender ces mutations du monde, se les approprier, les intégrer dans nos analyses et délibérations, nos jugements moraux, nos choix, nos pratiques professionnelles au quotidien. «Sur dix erreurs politiques, il y en a neuf qui consistent simplement à croire encore vrai ce qui a cessé de l'être», disait le philosophe Henri Bergson (1934). Être actrice ou acteur de changement, c'est mettre en délibération nos valeurs profondes, nos repères spirituels, culturels et historiques, et les enrichir des données que portent les mutations en cours du monde. Être actrice ou acteur de changement, c'est appartenir à des communautés de changement ou les faire émerger.

Nous sommes les contemporains, les témoins de la fin d'un long cycle de l'histoire de la famille humaine, cette longue période de colonisation, d'apartheid social, spirituel et culturel qui s'est étendue sur trois siècles d'une violence extrême portée aux confins du monde par les puissances européennes. Le basculement de la richesse et le déploiement universel de l'ère numérique ont notamment pour effet de donner aux cultures du monde, jusque-là méprisées, niées et enfouies, les moyens de leur résurrection, de leur affirmation et de leur rayonnement. La Chine, l'Inde, l'Indonésie, les États arabes du golfe Persique, le Nigéria, le Maroc, le Mexique, le Brésil sont aujourd'hui des puissances culturelles en expansion qui rayonnent partout dans le monde. Au moment où nous sommes réunis, la Chine est l'invitée du Salon du livre d'Alger et la Turquie de la foire culturelle de Kigali. Sur les plans financier, économique, commercial et culturel, les rapports entre pays du Sud avancent à vitesse grand $\mathrm{V}$ sur la place du monde. À titre d'exemple, depuis 20 ans, la Chine a créé 516 centres culturels dits instituts Confucius et 1706 salles Confucius dans 142 pays. Certes, l'Occident constitue toujours une zone de puissance, mais il n'est plus seul à diriger les institutions multilatérales et à fixer les normes pour tous. Ce temps est révolu.

D'autres institutions émergent, telle la Banque asiatique d'investissement pour les infrastructures, à laquelle 80 pays ont apporté leur appui à ce jour. D’autres visées de développement international se déploient, comme l'extraordinaire projet en cours de la nouvelle route de la soie, vaste plan d'infrastructure mondiale partant de la Chine et utilisant deux routes, l'une terrestre et l'autre maritime. Ce projet concernerait 65 pays, $62 \%$ de la population mondiale et
$31 \%$ de son PIB, ainsi que $40 \%$ des surfaces émergées. Il serait sans équivalent dans l'histoire contemporaine avec ses quelque 1000 milliards de dollars de budget. Peut-être faudrait-il y accorder une importance au moins égale à celle accordée au dernier tweet de Donald Trump?

Être acteur de changement pour les professionnels de l'information, c'est présenter aux Québécois le monde tel qu'il advient, en débattre, en montrer les nouvelles dynamiques, les enjeux et défis, les partager, leur faire une place dans nos délibérations, nos collections, nos médiations. Notre responsabilité est majeure tant la société que nous formons en Amérique et dans le monde a le besoin vital d'une information intègre pour ne pas se tromper de direction dans la formation de son capital humain, l'utilisation de son capital financier, ses choix en matière de recherche et développement, et ses partenariats internationaux. Collectivement, comme professionnels de l'information, nous sommes aux avant-postes de cette continuelle quête de données intègres et actualisées dont nos communautés ont besoin, données qui accompagnent le Québec et ont contribué à tracer la direction qu'il a empruntée et emprunte encore aujourd'hui. Ces chemins ont mené de la colonisation intérieure qui a ouvert les régions du Québec à la montée du syndicalisme et de l'économie coopérative qui nous a donné nos premières assises sociales, par le biais de la CSN, de Desjardins, de la Coop fédérée, de la création de nos grandes écoles, l'École des sciences sociales de l'Université Laval, Polytechnique et HEC Montréal, sans oublier la bibliothèque Saint-Sulpice, et jusqu'au Refus global toujours d'actualité qui nous a proposé une pensée de rupture; de l'appropriation de la télévision à la Révolution tranquille qui nous a donné un ministère de l'Éducation, Hydro-Québec, la Caisse de dépôt, une présence internationale propre, un réseau d'universités sur tout le territoire, la Charte des droits et libertés de la personne, la Charte de la langue française, la Paix des braves, le Québec Inc., le virage technologique largement inspiré par Bernard Landry qui a fait de Montréal, à terme, l'une des cinq ou six villes du monde où se pensent et se construisent l'aéronautique de l'avenir et un maillon solide dans la recherche et la production de l'intelligence artificielle et de ses applications. Montréal, comme vous le savez, accueillera, durant trois années de suite, de 2019 à 2021, le Sommet mondial sur l'intelligence artificielle.

Pour atteindre ces sommets, il aura fallu un savant montage d'initiatives publiques et privées, l'appui institutionnel des universités, la mise en convergence de grands projets de recherche, la formation de chercheurs et d'auxiliaires de recherche. Ainsi s'est accomplie la gestation de l'un des laboratoires les plus renommés au monde en intelligence artificielle, ainsi s'est constituée la communauté de l'intelligence artificielle de Montréal. La récolte de l'Institut des algorithmes d'apprentissage de Montréal est somptueuse, avec 
l'inauguration en 2017 de la branche canadienne du Facebook Artificial Intelligence Research ${ }^{3}$ (FAIR), l'installation par Google de deux équipes issues de sa filiale britannique Deepmind et de son laboratoire Google Brain, l'arrivée de Thales avec son laboratoire cortAIx, ainsi que de Microsoft et de sa jeune entreprise Maluuba, spécialisée dans le traitement des langues.

En amont de toutes les formidables réalisations que je viens d'évoquer, de la colonisation et de l'occupation de notre territoire physique à la maîtrise de l'intelligence humaine, il y a un besoin de produire de l'information jusqu'au point où l'information elle-même devient le matériau de l'expérimentation, la matière première de l'entreprise humaine qui croit pouvoir y insuffler une énergie vitale. Il y a aussi une conjugaison de trois communautés, une communauté de recherche, une communauté de savoir-partage et une communauté de volonté, ces composantes du changement dans une société démocratique. La géopolitique en mutation porte à un haut niveau ses exigences pour notre société, et en conséquence, pour celles et ceux qui ont vocation de la raccorder avec l'émergence continue de la connaissance. Troublant, angoissant et passionnant. La vie de la nation est liée à la connaissance qui la nourrit. C'est là que se situe la responsabilité sociale des professionnels de l'information. Nous sommes arrivés à ce qui commence, selon le superbe mot de Gaston Miron (1970).

Récemment, la directrice générale de la Grande Bibliothèque, notre amie Danielle Chagnon, évoquait la mise en valeur de collections en lien avec les objectifs de développement durable des Nations Unies, 17 objectifs pour sauver le monde ${ }^{4}$. Nous pourrions examiner dans le cadre de nos tables de concertation, comme cela a semble-t-il déjà été fait, la possibilité de fédérer certaines initiatives pour débattre de la nouvelle carte politique, économique et culturelle du monde et de sa signification pour nos institutions et leur offre. Nous pourrions aussi débattre de ce que nous pouvons faire ensemble pour apporter notre soutien à ces objectifs de développement durable. Délibérer, certes, mais aussi agir en espérant que cet engagement se conjuguera avec ceux du plus grand nombre.

Est-il raisonnable d'avoir une telle conviction, de croire que l'on peut ainsi infléchir la direction des flux abondants qui irriguent et sollicitent l'esprit humain? La liste des réalisations accomplies à diverses étapes de notre histoire que j'ai évoquée plus haut plaide pour une réponse positive. J'y ajouterai les avancées suivantes: le mouvement mondial pour l'égalité et l'équité des genres, pour la reconnaissance de la diversité sexuelle, pour la prise en compte de la

3. ici.radio-canada.ca/nouvelle/1125202/laboratoire-intelligenceartificielle-facebook-montreal-anniversaire-expansion-montreal

4. www.un.org/sustainabledevelopment/fr/objectifs-dedeveloppement-durable/ dégradation de notre planète, pour l'instauration de la Cour pénale internationale, pour la reconnaissance des droits des peuples autochtones, parmi bien d'autres. Ces projets ont d'abord vécu dans le cœur et l'esprit de quelques-uns, puis sont devenus le fait de collectifs restreints qui n'ont cessé de grandir, de se fédérer et de se doter de leviers leur permettant d'accéder finalement au statut de biens communs de l'humanité.

\section{Quels changements pour nous?}

À BAnQ, nous partageons avec vous les enjeux et défis que je viens d'énumérer. Comprendre et intégrer les changements du monde implique de porter une attention nouvelle à la pluralité des sources de la science, de la technologie, de la culture, du savoir. Ces sources s'universalisent progressivement: nos enfants ont déjà intégré en partie cette évolution, eux qui connaissent et apprécient les chanteurs de la K-pop coréenne, les stars du cinéma nigérian, les jeux vidéo avec leurs super-héros asiatiques, les œuvres du peintre Takashi Murakami, les mangas japonais, etc.

En clair, il nous faut prendre acte que le monde occidental n'est plus l'espace de référence, comme ce fut le cas ces trois derniers siècles. Il s'insère désormais et irrémédiablement dans un espace mondial qui multiplie les lieux d'innovation culturelle, sociale, scientifique et technologique. Les Émirats arabes unis sont devenus des plaques tournantes culturelles reconnues, la Chine, le leader mondial de la lutte environnementale, de l'usage d'Internet et de l'intelligence artificielle, le Maroc, une puissance africaine de premier plan. Tout cela doit se traduire par un impact, à terme, sur nos collections, nos recommandations et nos médiations. Cela implique pour nous de nous intéresser à cette communauté internationale nouvelle, et de nous familiariser avec les géographies spirituelle, économique et scientifique du monde, dont notre propre diversité actuelle et à venir constitue un fragment, notre fragment.

Notre institution, comme les vôtres, a vocation de permettre au plus grand nombre d'explorer ce monde nouveau. Cela nécessite une intervention soutenue pour faire reculer le scandale de l'illettrisme sous toutes ses formes. Il nous faut augmenter notre offre en ce sens, et avec vous toutes et tous, les écoles, les médias, faire de la lutte contre l'illettrisme une cause nationale. Le slogan «zéro illettrisme» doit être partagé par toutes les Québécoises et tous les Québécois. À BAnQ, nous nous sommes résolument engagés dans cette voie.

Cette exploration du Nouveau Monde, pour emprunter une expression chère aux grands voyageurs de la Renaissance, pourrait se traduire aussi par des ateliers spécifiques, des délibérations publiques, des expositions spécialisées, des échanges et des partenariats avec les institutions de ces 
pays que l'on dit émergents, mais qui dans de nombreux domaines ont dépassé ce stade depuis longtemps. Il nous faut saisir toutes les opportunités, et au besoin les créer quand elles font défaut, afin de nouer des liens sur objectifs avec les institutions des pays de la grande zone asiatique où se déplace à vitesse grand $\mathrm{V}$ la centralité politique et économique du monde. Nous devons mieux les connaître, tout comme ils doivent mieux nous connaître.

Le passage à la civilisation numérique sera pour tous long, complexe et exaltant. À BAnQ, il occupe et occupera une place centrale dans nos propres délibérations, investissements et propositions, pour la raison très simple que c'est là que se trouve notre public, en nombre sans cesse croissant, et que l'on peut compter en millions. Dans les missions et fonctions de notre institution comme Archives nationales, nous sommes engagés avec l'État québécois dans la mise en place de systèmes intégrés d'indexation numérique des documents, dès leur création jusqu'à leur dépôt dans nos archives, ce qui est la partie essentielle d'une administration publique numérique. Cette fonction-conseil que nous exerçons se double de la création par nos équipes d'archivistes et de techniciens du numérique de systèmes technologiques conséquents pour les 2150 ministères et organismes publics avec lesquels nous travaillons. Nous sommes aussi engagés avec l'État québécois dans la mise en place du dépôt légal numérique, mesure 44 du Plan culturel numérique et absolue nécessité pour un Québec accusant un retard considérable sur l'ensemble des pays de l'OCDE, y compris le Canada. Même si elle est difficile à estimer avec précision, une part substantielle de la création numérique québécoise, et donc du patrimoine documentaire québécois, échappe présentement au trésor national que constituent les collections patrimoniales enrichies en permanence par le dépôt légal. Nous espérons vivement que le nouveau gouvernement mettra fin à une situation aussi déplorable.

S'agissant de BAnQ comme institution fédératrice des trois grandes maisons que sont les Archives nationales, la Bibliothèque nationale et la Grande Bibliothèque du Québec, nous sommes engagés dans de grands chantiers qui, nous l'espérons, vont nous permettre de fournir à nos millions d'usagers une offre numérique de grande qualité. Des équipes s'affairent déjà à mettre à niveau notre intranet ainsi que notre portail Internet et à permettre l'accessibilité de l'offre numérique de BAnQ sur les plateformes mobiles, afin que cette dernière soit exemplaire. En parallèle, nous en sommes à numériser, comme il se doit, les vastes collections patrimoniales dont certaines demandent une véritable gestion de projets, plus qu'une gestion continue et prévisible des pièces qui témoignent de notre histoire nationale. Enfin, le dépôt numérique fiable des pièces numérisées fait l'objet de recherches intensives tant l'offre actuelle demeure inadéquate. Pour tout vous dire, nous en sommes aussi à revoir la gouvernance du numérique dans notre institution. Elle y occupera une place centrale dès le prochain exercice. Enfin, avec nos partenaires, la Ville de Montréal et le ministère de la Culture et des Communications, nous sommes à l'œuvre pour faire en sorte que la bibliothèque Saint-Sulpice, qui fut à l'avant-garde de la connaissance au début du siècle précédent, le soit à nouveau en ce début de siècle, cette fois au regard de la civilisation numérique.

Mais notre inclusion dans la civilisation numérique ne doit pas se faire aux dépens de notre tradition historique. L'humanité historique et l'humanité numérique doivent coexister: le temps, ce XXI ${ }^{\mathrm{e}}$ siècle, les départagera. Certains s'inquiètent des dérèglements actuels des systèmes d'information classique: déplacement massif de la publicité en direction de la Toile, doublement des éditions par ajout de la version numérique, abandon de l'édition papier, baisse des revenus de ces systèmes d'information, mises à pied, etc. Tous les médias traditionnels ou presque sont progressivement affectés par le déploiement de l'ère numérique. La période de transition est certes difficile, mais elle ouvre sur des compensations nouvelles: taxes nationales sur les revenus des multinationales du domaine, comme vient d'en décider l'Union européenne qui, par ailleurs, a légiféré pour assurer la protection de la vie privée sur la Toile; imposition spécifique pour les publicités sur les réseaux sociaux, mais aussi taxe sur les équipements comme on le fait en matière environnementale. Cet ensemble de ressources publiques devrait alimenter un fonds de la création auquel les équipes des systèmes d'information classiques auraient accès. Le reste dépend de la capacité et de la volonté de création des uns et des autres, du rassemblement de communautés d'intérêts, de la pertinence de l'offre par rapport aux besoins et attentes des citoyens.

Ces nouvelles dimensions de l'organisation de l'information affecteront aussi nos bibliothèques, dont les fonctions vont être progressivement transférées sur la Toile. À l'âge de la robotique, des algorithmes, de l'intelligence artificielle, des données de masse, quel est donc l'avenir de la mémoire, de la production et du partage de la connaissance? Quel est l'avenir de la création? Il nous faut maîtriser ces nouveaux leviers. À BAnQ, une équipe dresse les hypothèses d'utilisation des données de masse. Nous entendons organiser avec certains de nos partenaires la recherche, l'analyse, la délibération et la concertation autour des questions que soulèvent ces nouveaux leviers. Pour comprendre, pour entreprendre, pour réussir le changement qui est avéré, pour en faire l'occasion d'une offre encore mieux adaptée aux besoins de nos clientèles individuelle et collective, aux besoins du Québec. Je vous invite à la confiance.

À BAnQ, nous sommes en train de redessiner et d'enrichir notre offre éducative, en conjuguant les perspectives de l'éducation tout au long de la vie et de l'appui aux 
programmes scolaires. Nous avons l'ambition de développer une offre éducative vaste et unique, parce que découlant des collections patrimoniales. Au premier trimestre de 2019, notre offre aux nouveaux Québécois sera elle aussi renouvelée et enrichie, en proposant de leur montrer ce qu'est la société d'accueil, son histoire, ses réalisations, ses enjeux et défis. Enfin, toujours en 2019, nous viserons à développer et à enrichir notre offre actuelle aux personnes âgées, qui compteront pour le quart de notre population en 2040. Moins mobiles, les membres de ce groupe d'âge sont cependant des citoyens à part entière à qui nous devons un accès intégral aux services publics. Nous les atteindrons là où ils vivent. Ils ne peuvent pas, ne doivent pas être exclus de l'âme de la rencontre qui est l'essence même de notre service public. Nous menons actuellement et allons poursuivre dans un avenir proche des études majeures consacrées aux effets du vieillissement de la population sur les bibliothèques, et au meilleur usage des données de masse que nous détenons.

Pour durer et nous affirmer dans le système global qui dispose de moyens quasi illimités, il nous faut remplir trois conditions. Premièrement, maîtriser et utiliser les systèmes scientifiques et épistémologiques qui sont le vecteur d'une connaissance poussée de nos usagers, de leur environnement, de leurs besoins, de leurs désirs et de leurs choix. Deuxièmement, nous préparer aux bibliothèques de demain, lieux de partage organisationnel et fonctionnel entre intelligence naturelle et intelligence artificielle, ressources humaines et ressources robotiques, traduction classique et système automatique d'équivalence linguistique, collections historiques et masses dématérialisées, systèmes documentaires nationaux et index universels de connaissances. Ces «lab-bibliothèques » auront pour matériaux des flux considérables de données tirées des comportements et des pratiques humaines, et dont l'analyse produira des flux de connaissances nouvelles. Troisièmement, bâtir une offre de proximité, de qualité et d'échange, qui créera du lien, de la confiance et de la fidélité. Avec leurs immenses moyens, les multinationales du domaine ne pourront jamais créer une telle offre, même si les algorithmes fouillent jusqu'à nos ADN. David Lankes a mille fois raison: nous sommes de la communauté ou nous ne sommes pas ${ }^{5}$. Enfin, les actrices et acteurs de changement que nous sommes doivent veiller avec le plus grand soin au maintien et au renforcement de l'architecture éthique du monde, ce qui implique la prise en compte des droits de toutes et tous, y compris le droit inaliénable à la connaissance et au développement d'une conscience vive de la responsabilité humaine.

N’oublions pas: zéro illettrisme.

5. Voir notamment Expect More: Demanding Better Libraries For Today's Complex World (2012).

\section{SOURCES CONSULTÉES}

Bergson, H. (1934). La pensée et le mouvant. Paris, France: Éditions Félix Alcan.

Miron, G. (1970). L’homme rapaillé. Montréal, Québec: Presses de l'université de Montréal.

Organisation de coopération et de développement économiques (OCDE). (2010). Perspectives on Global Development 2010: Shifting Wealth. Paris, France: Les Éditions de l'OCDE.
Spinoza, B. (1955). Traité des autorités théologique et politique. Paris, France: Gallimard.

Woolf, V. (1937). Les vagues. Paris, France: Stock.

Yourcenar, M. (1980). Les yeux ouverts. Paris, France: Le Centurion. 